\title{
Interhemispheric Effect of Parietal TMS on Somatosensory Response Confirmed Directly with Concurrent TMS-fMRI
}

\author{
Felix Blankenburg, ${ }^{1,2,4}$ Christian C. Ruff, ${ }^{1,2}$ Sven Bestmann, ${ }^{2,3}$ Otto Bjoertomt, ${ }^{1}$ Neir Eshel, ${ }^{1}$ Oliver Josephs, ${ }^{2}$ \\ Nikolaus Weiskopf, ${ }^{2}$ and Jon Driver ${ }^{1,2}$ \\ ${ }^{1}$ Institute of Cognitive Neuroscience, University College London, London WC1 3AR, United Kingdom, ${ }^{2}$ Wellcome Trust Centre for Neuroimaging and \\ ${ }^{3}$ Sobell Department for Motor Neuroscience and Movement Disorders, Institute of Neurology, University College London, London WC1N 3BG, United \\ Kingdom, and ${ }^{4}$ Department of Neurology and Bernstein Center for Computational Neuroscience, Charité, 10115 Berlin, Germany
}

Transcranial magnetic stimulation (TMS) has been used to document some apparent interhemispheric influences behaviorally, with TMS over the right parietal cortex reported to enhance processing of touch for the ipsilateral right hand (Seyal et al., 1995). However, the neural bases of such apparent interhemispheric influences from TMS remain unknown. Here, we studied this directly by combining TMS with concurrent functional magnetic resonance imaging (fMRI). We applied bursts of $10 \mathrm{~Hz}$ TMS over right parietal cortex, at a high or low intensity, during two sensory contexts: either without any other stimulation, or while participants received median nerve stimulation to the right wrist, which projects to left primary somatosensory cortex (SI). TMS to right parietal cortex affected the blood oxygenation level-dependent signal in left SI, with high- versus low-intensity TMS increasing the left SI signal during right-wrist somatosensory input, but decreasing this in the absence of somatosensory input. This state-dependent modulation of SI by parietal TMS over the other hemisphere was accompanied by a related pattern of TMS-induced influences in the thalamus, as revealed by region-of-interest analyses. A behavioral experiment confirmed that the same right parietal TMS protocol of $10 \mathrm{~Hz}$ bursts led to enhanced detection of perithreshold electrical stimulation of the right median nerve, which is initially processed in left SI. Our results confirm directly that TMS over right parietal cortex can affect processing in left SI of the other hemisphere, with rivalrous effects (possibly transcallosal) arising in the absence of somatosensory input, but facilitatory effects (possibly involving thalamic circuitry) in the presence of driving somatosensory input.

Key words: TMS; fMRI; interhemispheric; rivalry; somatosensory; SI

\section{Introduction}

There are several precedents for suggesting that somatosensory processing can involve interhemispheric influences. Clinical disorders, such as somatosensory extinction or spatial neglect for the contralesional side of space (Vallar, 1997; Heilman, 2003), have often been discussed in terms of possible hemispheric "rivalry" (Kinsbourne, 1977; Oliveri et al., 1999b; Hilgetag et al., 2001; Heilman, 2003; Kobayashi et al., 2005; Dambeck et al., 2006). Neuroscience studies of the normal rather than damaged brain have produced some evidence for interhemispheric influences affecting somatosensory cortex, using both invasive methods in monkeys (Iwamura et al., 1994, 2001; Iwamura, 2000) and, more recently, neuroimaging of apparently transcallosal influences between primary sensory cortices (Staines et al., 2002; Hlushchuk and Hari, 2006; Tommerdahl et al., 2006; Devor et al., 2007; Kastrup et al., 2008).

\footnotetext{
Received July 1, 2008; accepted Aug. 14, 2008.

This work was supported by a Wellcome Trust Programme Grant to J.D., grants from the Medical Research Council (J.D., F.B., C.C.R.), the Bundesministerium für Bildung und Forschung (F.B.), and the Wellcome Trust (S.B.), and a Royal Society-Leverhulme Trust Senior Research Fellowship to J.D.

This article is freely available online through the J Neurosci Open Choice option.

Correspondence should be addressed to Felix Blankenburg, Department of Neurology and Bernstein Center for Computational Neuroscience, Charité, Philippstrasse 13, House 6, 10115 Berlin, Germany. E-mail: felix.blankenburg@charite.de.

DOI:10.1523/JNEUROSCI.3043-08.2008

Copyright $\odot 2008$ Society for Neuroscience $\quad$ 0270-6474/08/2813202-07\$15.00/0
}

A pioneering study on whether interhemispheric influences may affect somatosensory processing used transcranial magnetic stimulation (TMS) to show that TMS over right parietal cortex could enhance behavioral detection of ipsilateral right-hand somatosensory stimuli (Seyal et al., 1995). Although using only behavioral measures, the authors suggested that this finding may reflect interhemispheric influences analogous to those proposed on clinical grounds by Kinsbourne (1977). In discussion, Seyal et al. (1995) further noted that such interhemispheric modulation might involve thalamic gating, as suggested by Heilman (2003) in the clinical literature.

Although the study by Seyal et al. (1995) clearly established that TMS over right parietal cortex can affect somatosensory processing for right-hand inputs, the neural substrates for this could not be identified in that study, because the dependent measures were purely behavioral (Oliveri et al., 1999a; Seyal et al., 2005). Here, we aimed to study more directly the neural bases of any interhemispheric influences from right parietal TMS on somatosensory processing in the left hemisphere for unilateral righthand inputs, in close analogy to the classic study of Seyal et al. (1995), but now by combining TMS with concurrent functional magnetic resonance imaging (fMRI). Although the application of TMS during fMRI is methodologically challenging, it is now technically feasible and can allow direct study of any influences of local TMS on functional activity in remote but potentially inter- 
connected brain regions (Ruff et al., 2006, 2008; Bestmann et al., $2008 \mathrm{~b}$ ), including in the opposite hemisphere to that stimulated by TMS.

We used fMRI to measure brain responses during bursts of high-intensity (vs low-intensity) TMS over right parietal cortex, during the presence or absence of suprathreshold electrical stimulation of the right median nerve, which provides input to left primary somatosensory cortex (SI), i.e., opposite to the hemisphere stimulated here by TMS. We used short bursts of TMS at $10 \mathrm{~Hz}$ because this has proven effective in other recent concurrent TMS-fMRI studies from our lab (Bestmann et al., 2008b; Ruff et al., 2006, 2008). Our main interest was whether high (vs low) TMS over right parietal cortex would change brain responses in contralateral left SI. If the conjecture of Seyal et al. (1995) is correct, namely that right parietal TMS can enhance sensitivity of left somatosensory cortex to the presence versus absence of righthand somatosensory input, then we should expect the differential effect of right median nerve stimulation (vs no such somatosensory input) on the blood oxygenation level-dependent (BOLD) signal in left SI to become more pronounced during right parietal TMS. Also of interest was whether thalamic circuits might be affected, as we could study here with whole-brain fMRI.

\section{Materials and Methods}

Five healthy males (aged 27-36 years, right handed) with no history of neurological or psychiatric illness participated. All were screened for MRI and TMS compatibility and gave written informed consent in accord with local ethics. The study was approved by the joint ethics committee of the National Hospital for Neurology and Neurosurgery (University College London Hospitals National Heath Service Trust) and Institute of Neurology (University College London). Our stimulation protocol conformed to published TMS guidelines (Wassermann, 1998). The scalp position for placing the TMS coil over right parietal cortex was first determined outside the scanner, using the same approach as used by Seyal et al. (1995). For this purpose, we identified the motor hotspot for the left thenar muscles (motor threshold: mean $63.6 \pm 4.2 \%$ SEM of TMS stimulator output), and then moved the TMS coil backwards from the motor hotspot by $2-4 \mathrm{~cm}$ to the first point at which there was no longer any hand contractions induced when stimulating at the high TMS intensity of $110 \%$ of the resting motor threshold. We used an intensity of $50 \%$ of the motor threshold for our low-intensity TMS during the main combined TMS-fMRI experiment (see below). This motor threshold was determined inside the scanner room with the same equipment as used during scanning. Whereas some studies suggest that cross-modal interaction can have an impact on TMS thresholds (Ramos-Estebanez et al., 2007), our main focus for the concurrent TMS-fMRI experiment here was on any interaction between the effects of high versus low TMS during the presence or absence of concurrent tactile input to the other hemisphere (see Introduction). As described below, we found robust effects of TMS during scanning on somatosensory responses in the other hemisphere, thus confirming that our TMS protocol was effective.

A pair of surface-adhesive electrodes were positioned on the right wrist of the subject for median nerve stimulation. Constant current pulses (square wave, $200 \mu$ s duration) were applied to this site using a neurostimulator (DS7A, Digitimer) located within a shielded box (to preclude MR artifacts) inside the scanner room. Stimulation of the right median nerve in particular was confirmed by subjects' verbal report of sensation in the first three fingers. We ensured that the median nerve stimulation intensities used did not induce any twitching. For each subject, sensory threshold (mean, $2.4 \pm 0.16 \mathrm{~mA}$ ) was determined by the method of limits (single pulses lasting $200 \mu \mathrm{s}$ ), and stimulation intensity for the experiment was then set to three times that sensory threshold $(7.2 \pm 0.5 \mathrm{~mA})$, which is clearly detectable but does not induce any muscle effects. We used such suprathreshold somatosensory stimulation to ensure that increased activation of contralateral left SI by right median nerve stimulation could be reliably detected in fMRI (Arthurs et al., 2000; Blankenburg et al., 2003).
Our scanning experiment had a fully randomized $2 \times 2$ factorial design with the orthogonal factors of right parietal TMS (high vs low intensity) and right-wrist median nerve stimulation (present vs absent). Each trial consisted of three successive "mini-blocks" of stimulation, each lasting $500 \mathrm{~ms}$. On a random half of such trials, we applied right-wrist median nerve stimulation in three trains of five pulses (each at $10 \mathrm{~Hz}$ ). The other half of trials had no somatosensory stimulation. Orthogonally to this, TMS bursts (also five pulses at $10 \mathrm{~Hz}$, and thus similar to the combined TMS-fMRI protocols in previous studies from our lab) (Bestmann et al., 2008b; Ruff et al., 2006, 2008) were applied on each trial with either high or low TMS intensity (random half of trials each). On those $50 \%$ of trials in the $2 \times 2$ factorial design that had both TMS application and median nerve stimulation on the same trial, the trains of TMS or median nerve stimulation (both five pulses at $10 \mathrm{~Hz}$ ) were temporally interleaved with a $180^{\circ}$ difference in phase. Thus, each such trial started with a TMS pulse, followed $50 \mathrm{~ms}$ later by the first somatosensory stimulation, followed $50 \mathrm{~ms}$ later by the next TMS pulse, and so on. After each trial, a rest period without any stimulation (neither median nerve nor TMS) was included, lasting four image volumes.

Functional data were acquired on a $1.5 \mathrm{~T}$ whole-body scanner (Magnetom Sonata; Siemens), operating with the standard circular polarized receive- head and body-transmit coil. We used a multislice gradient echo echoplanar imaging sequence [39 slices; $64 \times 96$ matrix (readout $\times$ phase encoding); in-plane resolution, $3 \times 3 \mathrm{~mm}$; $2 \mathrm{~mm}$ slice thickness; $50 \%$ spatial gap between adjacent slices; echo time, $50 \mathrm{~ms}$; repetition time, $2880 \mathrm{~ms} ; 2298 \mathrm{~Hz} /$ pixel bandwidth; echo spacing, $500 \mu \mathrm{s}]$. In addition, oversampling $(50 \%)$ was used in the phase-encoding direction to shift any possible ghost artifact induced by mere presence of the TMS coil outside of the volume of interest. The last seven slices (33-39, lasting 630 $\mathrm{ms}$ ) were recorded without an MR excitation high-frequency pulse. This enabled us to apply the TMS pulses and the somatosensory stimulation always within this period, hence, without any potential corruption of functional image volumes. In addition, this ensured a constant auditory input from the scanner because gradients during these slices remained turned on. The acquisition time for one volume was $3.51 \mathrm{~s}$. Two sessions were acquired for each subject (each session comprised 320 volumes, including five dummy scans to allow T1 saturation). We chose to compare high-intensity (effective) TMS versus low-intensity (less effective or ineffective) TMS at a single right parietal site, similar to the site used by Seyal et al. (1995), rather than comparing different TMS sites because of the technical problems that relocating the TMS probe within an fMRI session would inevitably cause. Moreover, the appropriateness of high versus low TMS comparisons at a given site during concurrent fMRI was recently established by other studies from our group using similar concurrent TMS-fMRI protocols (Ruff et al., 2006; Bestmann et al., 2008b).

Each session included 44 randomly intermingled trials, 11 per condition in the $2 \times 2$ design. To preclude visual changes (e.g., from blinks), subjects kept their eyes closed throughout scanning. In addition, to ensure that TMS could not lead to any changes in performance that might otherwise have complicated interpretation of the physiological fMRI data, subjects had no behavioral task during scanning (Ruff et al., 2006). Thus, as per the Introduction, our a priori aim was to test for a physiological interaction of right parietal TMS with right-wrist somatosensory input, in terms of the BOLD response of left SI (and possibly thalamus as well) (see below). Nevertheless, we also ran a behavioral follow-up experiment outside the scanner (see below), which confirmed that our particular right parietal TMS protocol, using bursts at $10 \mathrm{~Hz}$, could indeed produce the same behavioral effect originally documented by Seyal et al. (1995), namely, enhancement of somatosensory detection on the ipsilateral right hand.

TMS during scanning was applied using a Magstim Super Rapid stimulator and an MR-compatible nonferrous figure-of-eight coil with a small diameter (30 mm inner diameter; $70 \mathrm{~mm}$ outer diameter; 15 turns each winding; wire size, $5 \times 1.5 \mathrm{~mm} ; 22.9 \mu \mathrm{H}$ inductance; $4.7 \mathrm{kVA}$ predicted maximal current at $100 \%$ ). The coil was positioned over the stimulation site tangentially to the scalp, at $\sim 45^{\circ}$ from the midline, inducing a biphasic current with an initial anteroposterior direction. We ensured that TMS did not induce any muscle twitches in the experiment (see above for TMS site selection). The coil was held fixed by a nonfer- 
romagnetic custom-built coil holder, and the participant's head was fixed with vacuum cushions. To avoid any radio-frequency interference of TMS with image acquisition, the TMS stimulator was placed in a shielded metal cabinet in the scanner room, and the TMS cable was passed through a custom filter box (Magstim) and additional ferrite sleeves (Wuerth Elektronik) (Ruff et al., 2006, 2008; Bestmann et al., 2008 b). Furthermore, the TMS coil was connected to the stimulator in parallel to a highvoltage relay (Magstim ES9486). During volume acquisition, this relay was closed, shorting any potential leaking current. Thus, any current flow through the stimulation coil originating from the stimulator was eliminated while it waited to release a pulse. The relay was opened $50 \mathrm{~ms}$ before a TMS train, and closed $11 \mathrm{~ms}$
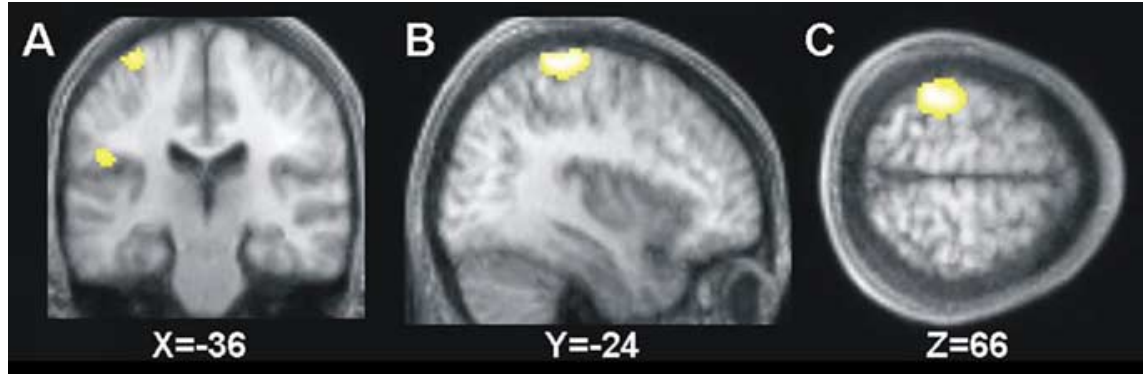

Figure 1. $\quad A-C$, Main effect of the presence minus absence of right-hand somatosensory stimulation (electrical stimulation of the right median nerve) shows highly significant activations ( $p<0.05$, FWE corrected) in contralateral SI (the dorsal activation visible in $\boldsymbol{A}-\boldsymbol{C}$ ) and SII (ventral activation visible here in $\boldsymbol{A}$ only). The activations are projected onto coronal $(\boldsymbol{A})$, sagittal $(\boldsymbol{B})$, and transverse $(\boldsymbol{C})$ slices of the averaged structural scans of all subjects. after the last TMS pulse of a train.

All stimuli were controlled using the Matlab (MathWorks) toolbox Cogent 2000 (http://www.vislab.ucl.ac.uk/Cogent/) running on a conventional PC. Image processing and analysis were performed with SPM2 (http://www.fil.ion.ucl.ac.uk/spm). Functional images were reconstructed off-line, and the first five images of each run discarded to avoid T1 equilibration effects. In accord with the standard statistical parametric mapping (SPM) approach, the remaining functional images were realigned to the first of the series, corrected for movement-induced image distortions, normalized to the Montreal Neurological Institute anatomical standard space, and spatially smoothed with a $9 \mathrm{~mm}$ full-width at half-maximum Gaussian kernel. In addition, the fMRI data were temporally bandpass filtered (lower and upper cutoff frequencies at 7 and $128 \mathrm{~s}$, respectively).

Statistical parametric maps were calculated by multiple regressions of the data onto a model of the hemodynamic response (Friston et al., 1995). This model contained regressors for the onsets of every miniblock for each of the four conditions in the $2 \times 2$ design, convolved with the canonical hemodynamic response function in SPM2. An autocorrelation model was used to account for scan-to-scan dependencies in the error term. Statistical inference used a fixed effect model, in accord with the limited number of subjects $(n=5)$ available for this demanding combined TMS-fMRI protocol. However, we also inspected individual data to ensure that the critical fMRI pattern was observed for all subjects (see below). For unrestricted whole-brain analyses, we used a threshold of $p<0.05$, familywise error (FWE) corrected for the entire image volume. For analyses of activity in brain areas for which we had clear a priori hypotheses (e.g., left somatosensory cortex and thalamus) (see Introduction), critical effects were inspected in volumes of interest (VOIs) derived either by anatomical criteria (e.g., the thalamus was defined by means of a computerized cytoarchitectonic atlas, see http://www.loni.ucla.edu/ ICBM/Downloads/Downloads_Atlases.shtml), or functionally by inclusive masking with an orthogonal contrast used to define specific brain areas of interest (e.g., activation for presence minus absence of righthand median nerve stimulation was used to confirm functional localization of contralateral left somatosensory cortex). The results of these hypothesis-driven analyses are all reported at a threshold of $p<0.05$, FWE corrected for the VOI (Worsley et al., 1996).

Here, we used bursts of TMS at $10 \mathrm{~Hz}$ during scanning to drive reliable BOLD responses by the TMS (Ruff et al., 2006, 2008; Bestmann et al., 2008b). Our particular TMS protocol thus differed from the original study by Seyal et al. (1995), which had used single-pulse TMS. Accordingly, we also conducted a new psychophysical study outside the scanner that sought to replicate the classical behavioral findings of Seyal et al. (1995), but now using the identical 10-Hz-burst TMS protocol as in our concurrent TMS-fMRI study. This follow-up behavioral experiment was conducted in four additional subjects who were again screened for TMS compatibility and gave written informed consent in accord with local ethics. Briefly, we applied five pulses of $10 \mathrm{~Hz}$ repetitive TMS at the outset of each trial, either at 110 or $50 \%$ of motor threshold, as during scanning. The TMS coil was again localized over right parietal lobe, using the iden-

tical procedure as for the main fMRI experiment. On a random half of these trials, perithreshold right median nerve stimulation (of the same duration and timing relative to TMS burst as for the fMRI experiment) was applied during TMS. On the other half of trials, TMS was applied in the absence of median nerve stimulation. After each trial, subjects were asked to respond by button-press whether right-hand tactile stimulation was present or not. Subjects each completed four blocks of 60 trials. Tactile stimulation intensity was determined separately for each block, with the aim of keeping the intensities perithreshold. Some blocks were removed from analysis because of $>90 \%$ accuracy (three blocks), a bias toward responding "absent" on $>90 \%$ of trials (two blocks), or a technical malfunction (two blocks). Nine blocks remained, yielding a total of 540 trials. Sensitivity $\left(d^{\prime}\right)$ and response bias (criterion) were calculated for each retained block, and paired $t$ tests were performed to determine the effect of TMS intensity on $d^{\prime}$ across blocks.

\section{Results}

\section{Concurrent TMS-fMRI experiment}

Main effect of right median nerve stimulation on BOLD signal We first considered BOLD signal changes caused by presence (minus absence) of median nerve stimulation on the right wrist, which would be expected to activate left somatosensory cortex and related regions. This contrast did indeed show the expected highly significant activation of contralateral left SI (peak at $x=$ $-38, y=-38, z=66)$ and also SII $(x=-50, y=-20, z=22)$ (Fig. 1). We also found some activation of the left thalamus caused by contralateral right median nerve stimulation, with a peak at $x=-4, y=-12, z=6$.

\section{Main effect of high versus low right parietal TMS}

The main effect of high- minus low-intensity TMS over right parietal cortex revealed activations very similar to those found in previous combined TMS-fMRI studies for such contrasts (Ruff et al., 2006, 2008; Bestmann et al., 2008b), mainly auditory cortices (peaks at $x=60, y=-14, z=12$, and $x=-58, y=-28, z=22$ ) because of the slightly louder "click" sound associated with higher-intensity TMS, as would be expected. More interestingly, we also found some activation of the thalamus (peak at $x=-10$, $y=-18, z=8$ ), a point we will return to. However, we did not find reliable BOLD signal changes within right parietal cortex, over which the TMS coil was placed. Previous concurrent TMSfMRI studies indicate that MR signal-to-noise ratio immediately under the TMS coil does not always allow BOLD effects to be uncovered there (Bestmann et al., 2008a)

\section{Interaction of high versus low right parietal TMS with presence} versus absence of right median nerve stimulation

Our main question of interest, given the previous study by Seyal et al. (1995), concerned the possible interaction between highversus low-intensity right parietal TMS and presence versus ab- 


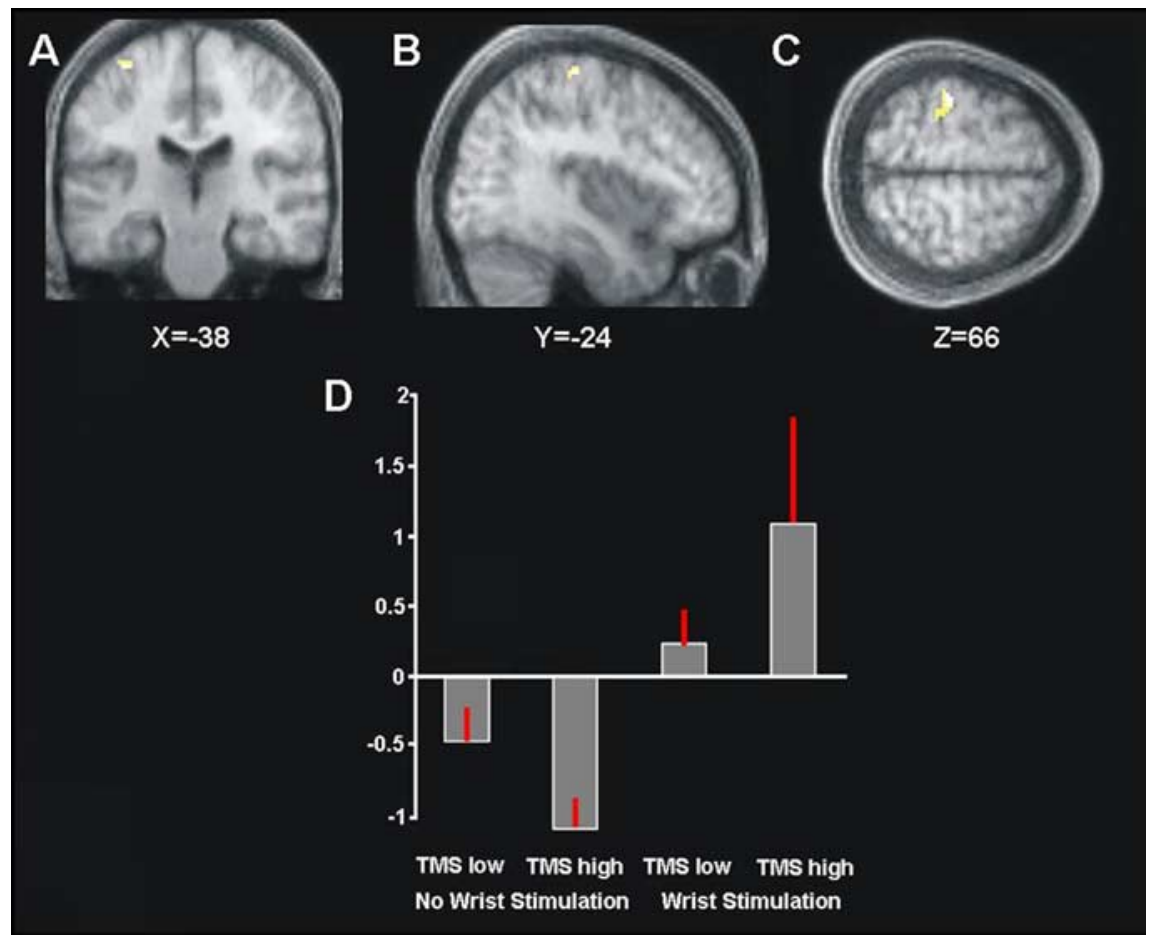

Figure 2. $\boldsymbol{A}-\boldsymbol{C}$, Areas showing an interaction between high- minus low-intensity right parietal TMS, and the presence minus absence of right median nerve sensory stimulation, within regions responding to right median nerve stimulation overall (compare Fig. 1). Significant ( $p<0.05$, FWE corrected) interaction effects are shown on coronal $(\boldsymbol{A})$, sagittal $(\boldsymbol{B})$, and transverse $(\boldsymbol{C})$ slices of the averaged structural scans. The left SI is clearly implicated. $\boldsymbol{D}$, Plot of the mean percentage of BOLD signal change extracted from the left SI cluster of the interaction contrast shown in $\boldsymbol{A}-\boldsymbol{C}$. High-versus low-intensity TMS over right parietal cortex increased the BOLD signal for left SI during right median nerve stimulation (compare third and fourth bars), but the same high TMS decreased the BOLD signal for left SI in the absence of somatosensory input (compare first and second bars). Restated, highintensity right parietal TMS increased the differential response of left SI to the presence versus absence of right-hand stimulation (compare fourth and second bars in the histogram), compared with low-intensity TMS (compare third and first bars).

sence of somatosensory input to the right median nerve that projects to contralateral left SI. In particular, as described in the Introduction, we aimed to test whether high versus low right parietal TMS could enhance the selective response of left somatosensory regions to the presence versus absence of right-wrist somatosensory input. This should be expressed as a specific interaction between TMS intensity and median nerve stimulation, namely, high minus low TMS during median nerve stimulation should lead to stronger BOLD increases than high minus low TMS without median nerve stimulation. To assess this for brain regions specifically responding to right median nerve stimulation, as per our hypothesis, we examined this interaction within a VOI representing the regions activated by median nerve stimulation (thresholded at $p<0.05$, FWE corrected) (Fig. 1). Please note that this VOI approach can only make our analyses more conservative, by requiring the reported regions to pass an additional test to confirm the specificity of their response pattern.

Within those brain regions already showing a main effect of presence minus absence of right median nerve stimulation (Fig. 1 ), we found an interaction of TMS intensity with presence/absence of median nerve stimulation for left SI (peaking at $x=-38$, $y=-24, z=64$ ) (Fig. $2 A-C$ ). The plot in Figure $2 D$ shows the mean percentage BOLD signal change in this left SI region (Fig. $2 A-C)$ for each of the four conditions. This plot shows that highversus low-intensity TMS over right parietal cortex enhanced the BOLD signal of left SI during the presence of right median nerve stimulation (Fig. 2D, compare fourth and rightmost bars with the third bar); whereas the same high versus low TMS over right parietal cortex tended instead to reduce BOLD signal in left SI when applied in the absence of right median nerve stimulation (compare second and first bars in plot of Fig. 2D). Note that this interaction was highly reliable across subjects, because the very same pattern was found for each of the five individual participants when inspecting their BOLD signal changes (mean coordinates of individual interaction peaks \pm SEM: $x=-36 \pm 2, y=-32 \pm 3$, $z=62 \pm 2$ ) within the left SI cluster that had responded to right median nerve stimulation in the group analysis (Fig. 1). Hence, our data indicate that the differential response of left SI to presence versus absence of right median nerve somatosensory input was significantly strengthened by high versus low right parietal TMS, thus providing a BOLD outcome that is logically analogous to the purely behavioral findings of Seyal et al. (1995); see also the results from our own behavioral follow-up study below using our $10-\mathrm{Hz}$-burst TMS protocol.

\section{Possible role of thalamus in interplay between right parietal TMS and left SI} Given that the thalamus had shown an effect in response to right median nerve stimulation [and had also been discussed by Seyal et al. (1995) as being potentially involved in interhemispheric effects of cortical TMS on somatosensory processing], we also conducted a VOI analysis of BOLD signal in the thalamus. For this purpose, we used a computerized cytoarchitectonic atlas (see Materials and Methods) to define anatomical VOIs corresponding to the thalami in both cortical hemispheres, and then applied a small-volume correction (Worsley et al., 1996) to assess any significant ( $p<0.05$, FWE corrected) interaction of TMS intensity and median nerve stimulation within these thalamic VOIs. This analysis revealed regions in the right and left thalamus (peaks at $x=10, y=-22, z=8$, and at $x=-12, y=-8, z=6$ ) (Fig. $3 A-C$ ) that showed similar activity changes to those found for left SI as reported above, albeit now primarily caused by a substantial increase in BOLD signal when high right parietal TMS was combined with right median nerve stimulation. Figure $3 D$ plots the corresponding percentage signal changes for the different conditions, confirming that BOLD signal in the left thalamus is boosted in the conjoint presence of right median nerve stimulation and high-intensity right parietal TMS. This pattern was also consistent across all participants (as confirmed by inspecting BOLD changes within the thalamic VOI at each individual's peak). Finally, Figure $3 E$ shows the equivalent group results for the right thalamus, i.e., now in the same hemisphere as the TMSstimulated right parietal cortex. Strikingly, this region also showed increased BOLD when high-intensity right parietal TMS was combined with right-hand somatosensory input (again consistently in all subjects, as confirmed by individual inspection). This finding suggests that bilateral thalami may start to influence each other interhemispherically, in the specific context of com- 
bined right-wrist somatosensory input and right parietal TMS, as part of a corticothalamic circuit influencing the two hemispheres.

\section{Behavioral follow-up experiment}

As predicted from our new BOLD findings, and consistent with the classic purely behavioral study by Seyal et al. (1995), our follow-up study found that our $10-\mathrm{Hz}-$ burst, right parietal TMS protocol significantly enhanced ipsilateral somatosensory detection, for perithreshold right median nerve stimulation. Sensitivity $\left(d^{\prime}\right)$, as calculated by signal detection theory, for this right-hand stimulation was significantly enhanced by high- versus low-intensity TMS at $10 \mathrm{~Hz}$, in a manner that was consistent across blocks (high-intensity TMS, $d^{\prime}=0.64$; low-intensity TMS, $d^{\prime}=0.18$; $\left.t_{(8)}=2.46, p=0.039\right)$. Sensitivity for highintensity TMS trials was significantly greater than zero $\left(t_{(8)}=4.05, p=0.0037\right)$, whereas the sensitivity on low-intensity TMS trials was not $\left(t_{(8)}=0.68, p=0.52\right)$. In contrast, TMS intensity did not affect response bias (high-intensity TMS, crite-
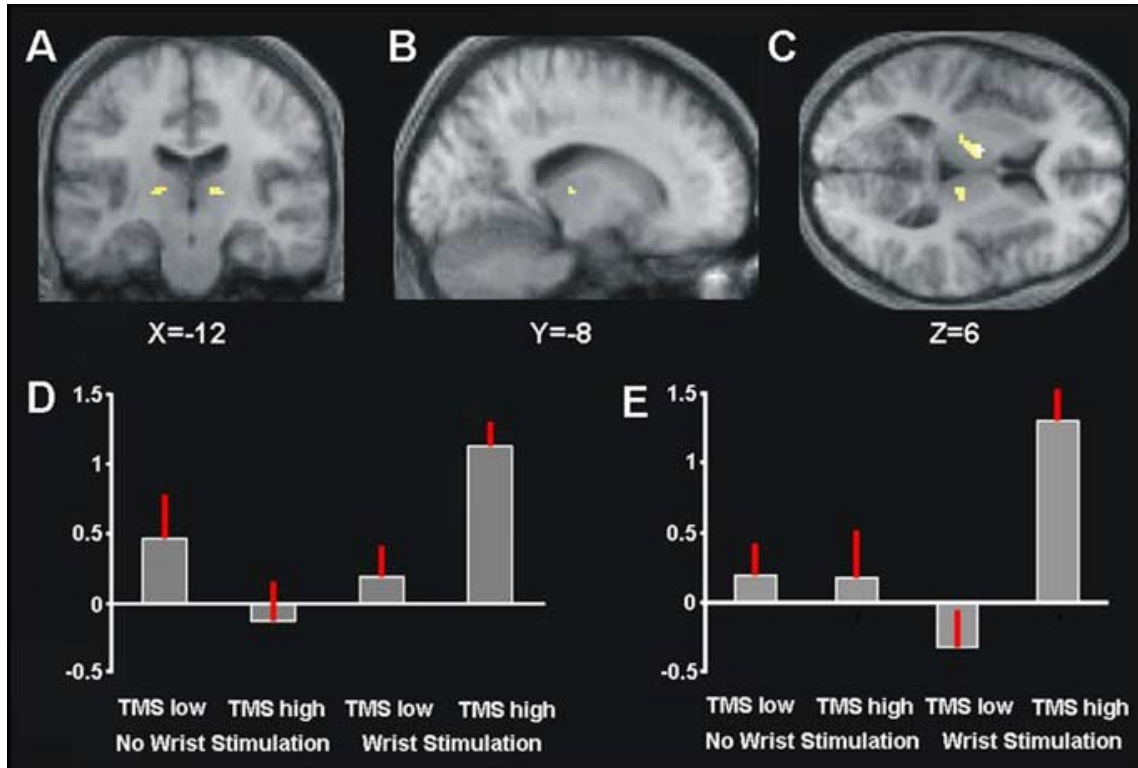

Figure 3. VOl analysis of the thalamus, as defined by a cytoarchitechtonic postmortem atlas (see Materials and Methods). $\boldsymbol{A}-\boldsymbol{C}$, Statistical parametric maps for the interaction of TMS and median nerve stimulation within the thalamus ( $p<0.05$, FWE corrected for that volume of interest) overlaid onto the mean structural scan. $\boldsymbol{D}, \boldsymbol{E}$, Plots of the group mean percentage of BOLD signal change from the left $(\boldsymbol{D})$ and right $(\boldsymbol{E})$ thalamic clusters for each condition in the interaction of TMS intensity and right-wrist stimulation. BOLD signal in the thalamus was highest during combined right-hand somatosensory stimulation and high-intensity TMS over right parietal cortex (fourth bar in each histogram).

rion $=0.21 ; t_{(8)}=-0.67, p=0.52$; neither criterion differed significantly from zero; both $p$ values $>0.49$ ). Thus, the same TMS protocol as we used during concurrent fMRI was shown to reproduce the classic behavioral finding by Seyal et al. (1995) of enhanced sensitivity for detecting ipsilateral right-hand touch.

\section{Discussion}

Although it is well accepted that somatosensory input projects contralaterally to SI, there has been increasing interest in possible interhemispheric interplay in somatosensory processing, both clinically (Oliveri et al., 1999b; Remy et al., 1999; Kobayashi et al., 2005; Sarri et al., 2006) and within basic neuroscience (Calford and Tweedale, 1990; Iwamura et al., 1994; Noachtar et al., 1997; Staines et al., 2002; Kanno et al., 2003; Nihashi et al., 2005; Hlushchuk and Hari, 2006; Tommerdahl et al., 2006; Devor et al., 2007; Kastrup et al., 2008). In a purely behavioral study, Seyal et al. (1995) demonstrated that TMS over right parietal cortex can benefit somatosensory processing for the ipsilateral (right) hand (Oliveri et al., 1999a; Seyal et al., 1997, 2005). In their classic study, Seyal et al. (1995) suggested that this may potentially reflect interhemispheric influences of right parietal TMS on left somatosensory cortex, perhaps arising transcallosally and/or via the thalamus. But because they used only behavioral measures, they could not directly ascertain the neural basis of possible interhemispheric TMS effects.

Here, we combined TMS with concurrent fMRI (Bestmann et al., 2008b; Ruff et al., 2006) to directly study the neural processes underlying interhemispheric effects of TMS to right parietal cortex. Like Seyal et al. (1995), we used an online TMS-approach (rather than off-line TMS), applying TMS during the presence or absence of somatosensory input to the ipsilateral hand, but now combining this with fMRI. We could therefore study the immediate impact of TMS on BOLD activity in SI of the opposite hemisphere, during the presence or absence of concomitant tactile input to that hemisphere. This allowed us to show directly that applying high- versus low-intensity TMS over right parietal cortex [with this TMS site selected similarly to that in the study by Seyal et al. (1995)] can indeed change the response of left SI to somatosensory input on the right hand. Specifically, such TMS enhanced the BOLD signal for left SI in response to right median nerve stimulation, although tending to have the opposite effect of reducing left SI BOLD signal when no right-hand stimulation was present (Fig. 2). This same interaction pattern was observed for each individual participant that we scanned. Another way of stating the interaction outcome is that the effect of presence versus absence of right-hand stimulation was enhanced for left SI during right parietal TMS, thus somewhat analogous to the finding of Seyal et al. (1995) that presence versus absence discrimination for right-hand touch could be enhanced by right parietal TMS. Moreover, in our behavioral follow-up study, we were able to replicate the finding by Seyal et al. (1995) of enhanced detection sensitivity for right-hand touch, during our high-intensity versus low-intensity right parietal TMS, using exactly the same $10 \mathrm{~Hz}$ TMS protocol that we had used during concurrent fMRI.

The interaction in the fMRI data for left SI here, in the hemisphere opposite to the right parietal TMS represents a "statedependent" remote physiological effect of TMS [for possible analogs in recent concurrent TMS-fMRI studies of the motor or visual systems, see the studies by Bestmann et al. (2008b) and Ruff et al. (2008), respectively]. Thus, the effect of local TMS applied to right parietal cortex on remote yet interconnected regions (such as left SI) depended here on the current context, i.e., whether or not somatosensory input was given concurrently to the right hand. There may be alternative physiological explanations for these observed remote effects, depending on whether TMS may have functionally disrupted activity in the right posterior parietal cortex (PPC) or increased cortical excitation under the TMS coil. On the latter possibility, the reduction in BOLD signal for left SI caused by right parietal TMS when applied in the absence of any concurrent somatosensory input to the right hand 
(Fig. 2D, second vs first bar) might potentially reflect interhemispheric inhibition that may arise transcallosally, as suggested for some other somatosensory paradigms, including invasive animal studies of trancallosal effects (Clarey et al., 1996) and more recent human neuroimaging studies (Hlushchuk and Hari, 2006; Devor et al., 2007; Kastrup et al., 2008). Such interhemispheric inhibition might potentially be mediated by stimulation of transcallosal fibers targeting GABAergic interneurons in the opposite hemisphere, as has been well established for the motor cortex (Daskalakis et al., 2002). Possible pathways for such an influence in the present experiment might be via transcallosal connections between Brodmann's areas 1 or 2, and/or motor cortex (Killackey et al., 1983).

The strikingly opposite pattern observed when applying the same right parietal TMS during right median nerve stimulation, with high-intensity right parietal TMS now enhancing BOLD signal for left SI in response to the somatosensory input to the right hand (Fig. $2 \mathrm{D}$ ), may relate to corticothalamic interplay [as already speculatively proposed by Seyal et al. (1995)]. When using a VOI analysis of the thalamus here, we found that the thalamus showed a somewhat similar interaction pattern to left SI (specifically with higher BOLD signal when high-intensity TMS was combined with the median nerve stimulation). Localization of this thalamic effect encompassing the ventroposterior nucleus (Fig. 3), which is known to respond to tactile stimulation (Malinen et al., 2006). However, some caution has to be applied when attributing BOLD signal changes to specific thalamic nuclei (such as tentatively for the ventroposterior nucleus here) because of anatomical and functional intersubject variability (Davis et al., 1998). Nevertheless, a number of studies have indicated that activation of SI (Ergenzinger et al., 1998; Li et al., 2005) and/or the PPC as one component of an attentional network (Crick, 1984) can lead to reactivation of the thalamus, either directly via relay neurons, or indirectly because of disinhibition by the nucleus reticularis. The latter is a net-like structure that enfolds the thalamus, comprising inhibitory neurons that can gate thalamic relays (Heilman, 2003). Animal tracing studies indicate reticuloreticularis connections between thalami in either hemisphere (Chen et al., 1992; Raos and Bentivoglio, 1993; Battaglia et al., 1994), potentially consistent with the bilateral thalamic pattern found here functionally. Interhemispheric effects on thalamic gating, driven by TMS stimulation of right parietal cortex when the thalamus is also receiving sensory input, may thus contribute to the interactions between hemispheres found when TMS is combined with somatosensory input, as in the present study.

Although our concurrent TMS-fMRI study found robust remote effects of right parietal TMS on BOLD signals elsewhere (specifically in contralateral SI and in bilateral thalamus, where state-dependent effects of TMS were found that interacted with the presence or absence of concurrent right-hand touch), we did not find any reliable BOLD signal changes under the TMS coil itself, in right parietal cortex. This particular outcome should be treated with some caution, because it is essentially a null result, and other recent TMS-fMRI studies have indicated that MR signal-to-noise ratio can sometimes be reduced immediately under the TMS coil (Bestmann et al., 2008a). But this does not undermine our clear effects on remote areas, including in the hemisphere opposite to TMS application, which clearly demonstrate an interhemispheric influence of the right parietal TMS. Although it might be interesting in future work to extend the paradigm we have introduced to address a more "extinction-like" situation with bilateral somatosensory inputs projecting to both hemisphere, for the present purposes of demonstrating a clear interhemispheric effect, it was a virtue to apply TMS to one hemisphere while stimulating the other unilaterally with somatosensory input. Moreover, in this respect, our study remained closely analogous to the classic study by Seyal et al. (1995), which likewise applied TMS to right parietal cortex while examining the impact for somatosensory inputs applied unilaterally to the ipsilateral hand.

In conclusion, our data confirm directly that TMS applied over one hemisphere [here, right parietal cortex, as in the study by Seyal et al. (1995)] can affect somatosensory responses in the other hemisphere (here, left SI). These remote effects of right parietal TMS apply in a manner that depends on concurrent somatosensory input to the right hand (ipsilateral to TMS), and may relate to corticothalamic circuitry as well as transcallosal connections. More generally, concurrent TMS-fMRI can show causal influences of local TMS on brain activity in remote but interconnected regions in the opposite hemisphere that can vary in a state-dependent manner.

\section{References}

Arthurs OJ, Williams EJ, Carpenter TA, Pickard JD, Boniface SJ (2000) Linear coupling between functional magnetic resonance imaging and evoked potential amplitude in human somatosensory cortex. Neuroscience 101:803-806.

Battaglia G, Lizier C, Colacitti C, Princivalle A, Spreafico R (1994) A reticuloreticular commissural pathway in the rat thalamus. J Comp Neurol 347:127-138.

Bestmann S, Ruff CC, Driver J, Blankenburg F (2008a) Concurrent TMS and fMRI: methods and current advances. In: Oxford handbook of transcranial magnetic stimulation. (Wassermann EM, Paus T, eds), pp 569592. Oxford: Oxford UP.

Bestmann S, Swayne O, Blankenburg F, Ruff CC, Haggard P, Weiskopf N, Josephs O, Driver J, Rothwell JC, Ward NS (2008b) Dorsal premotor cortex exerts state-dependent causal influences on activity in contralateral primary motor and dorsal premotor cortex. Cereb Cortex 18:1281-1291.

Blankenburg F, Taskin B, Ruben J, Moosmann M, Ritter P, Curio G, Villringer A (2003) A Imperceptible stimuli and sensory processing impediment. Science 299:1864.

Calford MB, Tweedale R (1990) Interhemispheric transfer of plasticity in the cerebral cortex. Science 249:805-807.

Chen S, Raos V, Bentivoglio M (1992) Connections of the thalamic reticular nucleus with the contralateral thalamus in the rat. Neurosci Lett 147:85-88.

Clarey JC, Tweedale R, Calford MB (1996) Interhemispheric modulation of somatosensory receptive fields: evidence for plasticity in primary somatosensory cortex. Cereb Cortex 6:196-206.

Crick F (1984) Function of the thalamic reticular complex: the searchlight hypothesis. Proc Natl Acad Sci U S A 81:4586-4590.

Dambeck N, Sparing R, Meister IG, Wienemann M, Weidemann J, Topper R, Boroojerdi B (2006) Interhemispheric imbalance during visuospatial attention investigated by unilateral and bilateral TMS over human parietal cortices. Brain Res 1072:194-199.

Daskalakis ZJ, Christensen BK, Fitzgerald PB, Roshan L, Chen R (2002) The mechanisms of interhemispheric inhibition in the human motor cortex. J Physiol 543:317-326.

Davis KD, Kwan CL, Crawley AP, Mikulis DJ (1998) Functional MRI study of thalamic and cortical activations evoked by cutaneous heat, cold, and tactile stimuli. J Neurophysiol 80:1533-1546.

Devor A, Tian P, Nishimura N, Teng IC, Hillman EM, Narayanan SN, Ulbert I, Boas DA, Kleinfeld D, Dale AM (2007) Suppressed neuronal activity and concurrent arteriolar vasoconstriction may explain negative blood oxygenation level-dependent signal. J Neurosci 27:4452-4459.

Ergenzinger ER, Glasier MM, Hahm JO, Pons TP (1998) Cortically induced thalamic plasticity in the primate somatosensory system. Nat Neurosci $1: 226-229$.

Friston KJ, Holmes AP, Poline JB, Grasby PJ, Williams SC, Frackowiak RS, Turner R (1995) Analysis of fMRI time-series revisited. Neuroimage 2:45-53.

Heilman KM (2003) Clinical neurophysiology. Oxford: Oxford UP.

Hilgetag CC, Théoret H, Pascual-Leone A (2001) Enhanced visual spatial 
attention ipsilateral to rTMS-induced "virtual lesions" of human parietal cortex. Nat Neurosci 4:953-957.

Hlushchuk Y, Hari R (2006) Transient suppression of ipsilateral primary somatosensory cortex during tactile finger stimulation. J Neurosci 26:5819-5824.

Iwamura Y (2000) Bilateral receptive field neurons and callosal connections in the somatosensory cortex. Philos Trans R Soc Lond B Biol Sci 355:267-273.

Iwamura Y, Iriki A, Tanaka M (1994) Bilateral hand representation in the postcentral somatosensory cortex. Nature 369:554-556.

Iwamura Y, Taoka M, Iriki A (2001) Bilateral activity and callosal connections in the somatosensory cortex. Neuroscientist 7:419-429.

Kanno A, Nakasato N, Hatanaka K, Yoshimoto T (2003) Ipsilateral area 3b responses to median nerve somatosensory stimulation. Neuroimage 18:169-177.

Kastrup A, Baudewig J, Schnaudigel S, Huonker R, Becker L, Sohns JM, Dechent P, Klingner C, Witte OW (2008) Behavioral correlates of negative BOLD signal changes in the primary somatosensory cortex. Neuroimage 41:1364-1371.

Killackey HP, Gould HJ 3rd, Cusick CG, Pons TP, Kaas JH (1983) The relation of corpus callosum connections to architectonic fields and body surface maps in sensorimotor cortex of new and old world monkeys. J Comp Neurol 219:384-419.

Kinsbourne M (1977) Hemi-neglect and hemisphere rivalry. Adv Neurol 18:41-49.

Kobayashi M, Takeda K, Kaminaga T, Shimizu T, Iwata M (2005) Neural consequences of somatosensory extinction: an fMRI study. J Neurol 252:1353-1358.

Li L, Rema V, Ebner FF (2005) Chronic suppression of activity in barrel field cortex downregulates sensory responses in contralateral barrel field cortex. J Neurophysiol 94:3342-3356.

Malinen S, Schürmann M, Hlushchuk Y, Forss N, Hari R (2006) Improved differentiation of tactile activations in human secondary somatosensory cortex and thalamus using cardiac-triggered fMRI. Exp Brain Res 174:297-303.

Nihashi T, Naganawa S, Sato C, Kawai H, Nakamura T, Fukatsu H, Ishigaki T, Aoki I (2005) Contralateral and ipsilateral responses in primary somatosensory cortex following electrical median nerve stimulation-an fMRI study. Clin Neurophysiol 116:842-848.

Noachtar S, Lüders HO, Dinner DS, Klem G (1997) Ipsilateral median somatosensory evoked potentials recorded from human somatosensory cortex. Electroencephalogr Clin Neurophysiol 104:189-198.

Oliveri M, Rossini PM, Pasqualetti P, Traversa R, Cicinelli P, Palmieri MG, Tomaiuolo F, Caltagirone C (1999a) Interhemispheric asymmetries in the perception of unimanual and bimanual cutaneous stimuli. A study using transcranial magnetic stimulation. Brain 122: 1721-1729.

Oliveri M, Rossini PM, Traversa R, Cicinelli P, Filippi MM, Pasqualetti P, Tomaiuolo F, Caltagirone C (1999b) Left frontal transcranial magnetic stimulation reduces contralesional extinction in patients with unilateral right brain damage. Brain 122:1731-1739.

Ramos-Estebanez C, Merabet LB, Machii K, Fregni F, Thut G, Wagner TA, Romei V, Amedi A, Pascual-Leone A (2007) Visual phosphene perception modulated by subthreshold crossmodal sensory stimulation. J Neurosci 27:4178-4181.

Raos V, Bentivoglio M (1993) Crosstalk between the two sides of the thalamus through the reticular nucleus: a retrograde and anterograde tracing study in the rat. J Comp Neurol 332:145-154.

Remy P, Zilbovicius M, Degos JD, Bachoud-Lévi AC, Rancurel G, Cesaro P, Samson Y (1999) Somatosensory cortical activations are suppressed in patients with tactile extinction: a PET study. Neurology 52:571-577.

Ruff CC, Blankenburg F, Bjoertomt O, Bestmann S, Freeman E, Haynes JD, Rees G, Josephs O, Deichmann R, Driver J (2006) Concurrent TMSfMRI and psychophysics reveal frontal influences on human retinotopic visual cortex. Curr Biol 16:1479-1488.

Ruff CC, Bestmann S, Blankenburg F, Bjoertomt O, Josephs O, Weiskopf N, Deichmann R, Driver J (2008) Distinct causal influences of parietal versus frontal areas on human visual cortex: evidence from concurrent TMS fMRI. Cereb Cortex 18:817-827.

Sarri M, Blankenburg F, Driver J (2006) Neural correlates of crossmodal visual-tactile extinction and of tactile awareness revealed by fMRI in a right-hemisphere stroke patient. Neuropsychologia 44:2398-2410.

Seyal M, Ro T, Rafal R (1995) Increased sensitivity to ipsilateral cutaneous stimuli following transcranial magnetic stimulation of the parietal lobe. Ann Neurol 38:264-267.

Seyal M, Siddiqui I, Hundal NS (1997) Suppression of spatial localization of a cutaneous stimulus following transcranial magnetic pulse stimulation of the sensorimotor cortex. Electroencephalogr Clin Neurophysiol 105:24-28.

Seyal M, Shatzel AJ, Richardson SP (2005) Crossed inhibition of sensory cortex by $0.3 \mathrm{~Hz}$ transcranial magnetic stimulation of motor cortex. J Clin Neurophysiol 22:418-421.

Staines WR, Graham SJ, Black SE, McIlroy WE (2002) Task-relevant modulation of contralateral and ipsilateral primary somatosensory cortex and the role of a prefrontal-cortical sensory gating system. Neuroimage 15:190-199.

Tommerdahl M, Simons SB, Chiu JS, Favorov O, Whitsel BL (2006) Ipsilateral input modifies the primary somatosensory cortex response to contralateral skin flutter. J Neurosci 26:5970-5977.

Vallar G (1997) Spatial frames of reference and somatosensory processing: a neuropsychological perspective. Philos Trans R Soc Lond B Biol Sci 352:1401-1409.

Wassermann EM (1998) Risk and safety of repetitive transcranial magnetic stimulation: report and suggested guidelines from the International Workshop on the Safety of Repetitive Transcranial Magnetic Stimulation, June 5-7, 1996. Electroencephalogr Clin Neurophysiol 108:1-16.

Worsley KJ, Marrett S, Neelin P, Vandal AC, Friston KJ, Evans AC (1996) A unified statistical approach for determining significant signals in images of cerebral activation. Hum Brain Mapp 4:58-73. 